\title{
Determination of proper motions and membership of the open clusters NGC 1817 and NGC 1807`
}

\author{
L. Balaguer-Núñez ${ }^{1,2}$, K.P. Tian ${ }^{1}$, and J.L. Zhao ${ }^{1,3}$ \\ 1 Shanghai Astronomical Observatory, CAS Shanghai 200030, PR China \\ 2 Departament d'Astronomia i Meteorologia, Universitat de Barcelona, Avda. Diagonal, 647, E-08028 Barcelona, Spain \\ 3 CCAST(World Laboratory) P.O. Box 8730, Beijing 100080, PR China
}

Received January 5; accepted June 23, 1998

\begin{abstract}
Relative proper motions, their corresponding observed errors and membership probabilities of 722 stars in the old open cluster NGC 1817 region are determined from PDS measurements of 12 plate pairs. The plates have the maximum epoch difference of 81 years and were taken with the double astrograph at Zǒ-Sè station of Shanghai Observatory, which has an aperture of $40 \mathrm{~cm}$, a focal length of $6.9 \mathrm{~m}$ and a plate scale of $30^{\prime \prime} / \mathrm{mm}$. The average proper motion accuracy is $1.6 \mathrm{mas} / \mathrm{yr}$. These proper motions are used to determine the membership probabilities of stars in the region. The existence of two clusters in the region (NGC 1817, NGC 1807) is discussed. The number of stars with membership probability higher than 0.7 is 416 for NGC 1817 and 14 for NGC 1807.
\end{abstract}

Key words: open clusters — NGC 1807; NGC 1817 astrometry

\section{Introduction}

The open cluster NGC 1817 , in Taurus $\left[\alpha_{1997}=5^{\mathrm{h}} 11^{\mathrm{m}} 22^{\mathrm{s}}\right.$, $\left.\delta_{1997}=16^{\circ} 41^{\prime} 34^{\prime \prime}\right]$, is a rich, unstudied and presumably old open cluster (Janes \& Adler 1982; Phelps et al. 1994; Janes \& Phelps 1994). To date, the only photometric study of NGC 1817 was performed by Harris \& Harris (HH 1997) who obtained $U B V$ photometry to a limit $V=16.7$, of 265 stars in the central area of this cluster. A preliminary photometric study of the region by Purgathofer $(1961,1964)$ reached only to $V=14$. Previously, Cuffey (1938) obtained extensive photographic photometry of stars in this area in the blue and red bands to a limiting magnitude

Send offprint requests to: K.P. Tian or L. Balaguer-Núnez

* Table 5 is only available in electronic form from CDS via anonymous ftp 130.79.128.5, and by e-mail request to lbalague@pchpc3.am.ub.es of $R=14$. The location of the cluster at $1800 \mathrm{pc}$ almost directly in the Galactic anticenter direction and $400 \mathrm{pc}$ below the plane $[l=186.1, b=-13.1]$, and its low metallicity, make it an object of special interest for the research of the structure and the chemical evolution of the Galaxy.

Very little is known about the open cluster NGC 1807. Some authors considered it as not physical open cluster (Becker \& Fenkart 1971) or as possible member of multiple system with NGC 1817, 2244 and 2252 (Barkhatova 1963). Some of its G and K stars have DDO observations (Yoss et al. 1981; Piatti et al. 1995).

Proper motions of some stars in this region were published by Li (1954). But the accuracy of its proper motions is rather low since the epoch difference of the plates he used was only twelve years. Later on, in 1980's, three pairs of plates of NGC 1817 taken with the $40 \mathrm{~cm}$ astrograph at Shanghai Zǒ-Sè station, were measured manually on the Zeiss Ascorecord measuring machine at Zǒ-Sè. Relative proper motions in the cluster region of NGC 1817 were obtained from plate measurements, and its membership determination was made (Tian et al. 1983). However, these proper motions are not very accurate because of manual measuring.

In this paper we will, for the first time, determine accurate relative proper motions of 722 stars within a $1.5 \times 1.5$ area in the NGC 1817 region, from automatic measurements of 24 plates, six of which are newly taken. Through estimating membership probabilities with an improved likelihood method (Tian et al. 1998), the membership determination will lead us to distinguish two separate cluster in the region, with different distribution parameters: NGC 1817 and NGC 1807. 
Table 1. Plate material

\begin{tabular}{|c|c|c|c|c|c|}
\hline $\begin{array}{l}\text { Pairs } \\
\text { No. }\end{array}$ & plates & $\begin{array}{c}\text { Epoch } \\
(1900+)\end{array}$ & $\begin{array}{l}\text { Exp. Time } \\
\text { min }\end{array}$ & $\begin{array}{c}\text { Baselines } \\
\text { years }\end{array}$ & $\begin{array}{l}\text { No. of } \\
\text { Stars }\end{array}$ \\
\hline \multirow[t]{2}{*}{1} & CL61010 & 61.02 .02 & 40 & 21.06 & 693 \\
\hline & CL82001 & 82.02 .24 & 15 & & \\
\hline \multirow[t]{2}{*}{2} & CL415 & 16.01 .31 & 30 & 80.95 & 129 \\
\hline & P9704 & 97.01 .11 & 30 & & \\
\hline \multirow[t]{2}{*}{3} & CL5293 & 30.02 .16 & - & 52.02 & 610 \\
\hline & CL82003 & 82.02 .24 & 12 & & \\
\hline \multirow[t]{2}{*}{4} & CL54002 & 54.01 .31 & 80 & 28.07 & 620 \\
\hline & CL82006 & 82.02 .27 & 15 & & \\
\hline \multirow[t]{2}{*}{5} & CL54003 & 54.02 .08 & 90 & 28.05 & 568 \\
\hline & CL82004 & 82.02 .27 & 8'15 & & \\
\hline \multirow[t]{2}{*}{6} & CL5292 & 43.02 .22 & - & 37.84 & 525 \\
\hline & CL81011 & 81.12 .25 & 15 & & \\
\hline \multirow[t]{2}{*}{7} & CL61003 & 61.02 .12 & 20 & 35.92 & 541 \\
\hline & P9705 & 97.01 .12 & 30 & & \\
\hline \multirow[t]{2}{*}{8} & CL5291 & 43.02 .09 & - & 53.92 & 457 \\
\hline & P9706 & 97.01 .12 & 30 & & \\
\hline \multirow[t]{2}{*}{9} & CL61002 & 61.02 .17 & 30 & 21.02 & 672 \\
\hline & CL82002 & 82.02 .24 & - & & \\
\hline \multirow[t]{2}{*}{10} & CL81012 & 81.12 .25 & 15 & 15.05 & 408 \\
\hline & P9703 & 97.01 .11 & 30 & & \\
\hline \multirow[t]{2}{*}{11} & CL81010 & 81.12 .24 & - & 15.02 & 381 \\
\hline & P9701 & 97.01 .02 & 30 & & \\
\hline \multirow[t]{2}{*}{12} & CL81009 & 81.12 .24 & - & 15.04 & 335 \\
\hline & P9702 & 97.01 .11 & 30 & & \\
\hline
\end{tabular}

\section{Plate measurements and reduction of proper motions}

\subsection{Plate material and measurements}

In the present study 24 plates of the NGC 1817 region are available. They were taken with the double astrograph at the Zǒ-Sè station of Shanghai Observatory. This telescope, built by Gaultier in Paris at the beginning of this century, has an aperture of $40 \mathrm{~cm}$, a focal length of $6.9 \mathrm{~m}$, and a plate scale of $30^{\prime \prime} / \mathrm{mm}$. The size of the plates is 24 by $30 \mathrm{~cm}$, or $2^{\circ} .0 \times 2^{\circ} .5$. The oldest plate was taken in 1916 , and the newest ones in 1997 . The relevant information on these plates is given in Table 1 . The hour angles are not provided in Table 1 because the starting time of the first-epoch plates was not recorded.

All the plates were measured on a Photometric Data Systems (PDS) model 1010 automatic measuring machine at the Purple Mountain Observatory in Nanjing (China). There is a total of 916 stars being measured with a photographic magnitude limit close to about 15.0. An aperture size of 20 by 20 microns, scanning step of $20 \mu \mathrm{m}$, scanning speed of $25 \mu \mathrm{m} / \mathrm{s}$ and $R$ scanning type were adopted. The program for digital image centering is based on the algorithm developed by Lee \& Van Altena (1983). In order to monitor and reduce any possible plate displacements and the resulting systematic errors, all the stellar images to be measured were divided into 18 groups, all the groups sharing four common images which were scanned twice, before and after all the other images for each group. A Gaussian fit to the density array marginal distribution $F(x, y)$, was made following the software developed by Wang et al. (1995), from which the image center coordinates $\left(x_{0}, y_{0}\right)$, the background density $b$, peak density $D_{0}$, and the image radius $R$ can be obtained:

$F(x, y)=D_{0} \exp \left(\frac{-r^{2}}{2 R^{2}}\right)+b$

$r^{2}=\left(x-x_{0}\right)^{2}+\left(y-y_{0}\right)^{2}$.

\subsection{Proper motions}

The reduction of the relative proper motions for 722 stars in the region of NGC 1817 and NGC 1807 was made on the basis of the PDS measurements by means of an approach we have adopted many times before (Tian et al. 1982, 1983; Zhao et al. 1993, 1980; Su et al. 1998). There are three steps in the whole process: the first is to transform the measured results for all the plates to a common system, in order to eliminate the errors due to small differences in the orientation of different plates in scanning; the second step is to establish a reference frame, i.e. to decide upon the reference stars; the last step is to calculate proper motions of all the stars with respect to this reference frame, and their corresponding uncertainties.

After two loops of the least-squares adjustment, 83 stars with residuals in both $x$ and $y$ coordinates less than $2 \sigma_{x}$ and $2 \sigma_{y}$ respectively were chosen to be reference stars from the 99 stars common to all the plate pairs, where $\sigma_{x}$ and $\sigma_{y}$ are the rms residuals in the $x$ and $y$ coordinates obtained from the least-squares adjustment. This defines a preliminary proper motion system in which the proper motions of the selected stars are collectively free of translation, rotation and expansion.

Owing to the limited number of reference stars and the accuracies of the proper motions of these stars, the plate pair technique is used in the present study. All the linear and quadratic coordinate-dependent terms and the coma term are included in the plate solutions. The weighted mean of the proper motion of a star obtained from all of the available plate pairs should be taken as the final value of the proper motion of the star. The proper motion weight for a star in a plate pair is determined from the epoch difference of the pair and the measuring accuracy of the stellar image.

Table 2 gives the accuracies of final proper motions for stars in the NGC 1817 region with different numbers of measured pairs (greater than 2) and different distances from the field center. The units of the proper motions and their accuracies in this paper are mas/year. It is shown from the table that the accuracies depend strongly on the number of plate pairs, and the greater the number of pairs, the higher the accuracies of the final proper motions of the stars. This shows that increasing the number of available 
Table 2. Accuracies of proper motions for stars in different numbers of plate pairs and at different distances from the plate center in the NGC 1817/1807 region. Units are mas/yr

\begin{tabular}{ccccccccccccc}
\hline pairs & \multicolumn{4}{c}{$r \leq 15^{\prime}$} & \multicolumn{4}{c}{$15^{\prime}<r \leq 30^{\prime}$} & \multicolumn{3}{c}{$30^{\prime}<r \leq 45^{\prime}$} \\
\hline & $N$ & $\epsilon_{x}$ & $\epsilon_{y}$ & $\epsilon$ & $N$ & $\epsilon_{x}$ & $\epsilon_{y}$ & $\epsilon$ & $N$ & $\epsilon_{x}$ & $\epsilon_{y}$ & $\epsilon$ \\
$3-6$ & 28 & 2.16 & 2.02 & 2.09 & 83 & 2.17 & 2.04 & 2.11 & 32 & 1.78 & 1.82 & 1.80 \\
$7-9$ & 45 & 1.93 & 1.91 & 1.92 & 114 & 1.45 & 1.40 & 1.43 & 32 & 1.33 & 1.50 & 1.42 \\
$10-12$ & 146 & 0.89 & 0.86 & 0.88 & 158 & 0.85 & 0.77 & 0.81 & 23 & 0.94 & 0.98 & 0.96 \\
\hline $3-12$ & 219 & 1.38 & 1.33 & 1.35 & 355 & 1.44 & 1.37 & 1.41 & 87 & 1.43 & 1.52 & 1.48 \\
\hline
\end{tabular}

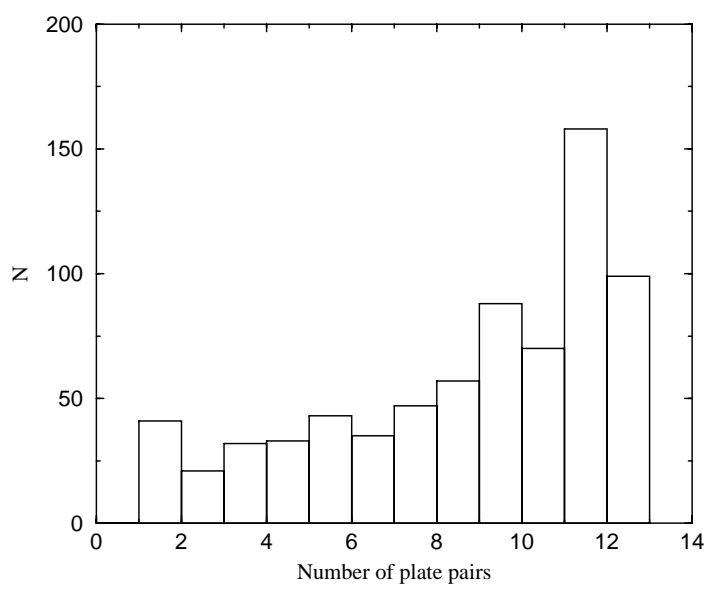

Fig. 1. The number of stars vs. the number of available plate pairs

plate pairs is very important for improving the accuracy of proper motions.

It can also be seen from the table that there is no obvious relation between the accuracies of the final proper motions and the distances of stars from the plate center, which shows that the imaging quality of the telescope has been very good and that the PDS machine was quite stable.

Figure 1 gives the number of stars for which different numbers of plate-pairs are available. More than $70 \%$ of proper motions are obtained from more than 6 plate pairs.

The rms errors of the proper motions of all 722 stars are $\epsilon_{x}= \pm 1.42 \mathrm{mas} / \mathrm{yr}, \epsilon_{y}= \pm 1.37 \mathrm{mas} / \mathrm{yr}$, and $\epsilon= \pm$ $1.39 \mathrm{mas} / \mathrm{yr}$, where $\epsilon=\sqrt{\frac{\epsilon_{x}^{2}+\epsilon_{y}^{2}}{2}}$. In the most accurate case, the rms errors are found to be 0.81 mas/yr for stars with 10 to 12 plates and distances to the centre between $15^{\prime}$ and $30^{\prime}$.

The rms proper-motions errors can be seen from Fig. 2, which shows the relations $N$ versus $\epsilon_{x}, N$ versus $\epsilon_{y}$ and $N$ versus $\epsilon$. So what we can say is that the accuracies of the proper motions of stars in the region of NGC 1817 and NGC 1807 obtained by us are relatively high, because of the good stellar images taken with the $40 \mathrm{~cm}$ double astrograph and the excellent positioning behavior of the PDS scanning machine.

\section{Membership determination}

Accurate membership determination is of fundamental importance for further astrophysical studies of clusters. The fundamental mathematical model set up by Vasilevskis et al. (1958) and the technique based upon the maximum likelihood principle developed by Sanders (1971) have been refined continuously since then.

An improved method for membership determination of stellar clusters based on proper motions with different observed accuracies was developed by Stetson (1980) and Zhao \& He (1990). Then Zhao \& Shao (1994) added the correlation coefficient of the field star distribution to the set of parameters describing their distribution on the sky. The spatial distribution of cluster stars and the dependence of the distribution parameters on the magnitudes of stars were considered by Su et al. (1998). Zhao et al. (1988, 1994) developed a statistical method on the same principle to determine the distribution parameters and membership of rich galaxy clusters. Shao \& Zhao (1996) then extended the above method to the situation of multiple substructures and multiple criteria, and developed a strict, rigorous, and useful mathematical model. Tian et al. (1998) adapted this multi-substructure and multi-criterion maximum likelihood method in one-dimensional radial velocity to the case of two dimensional velocity space (relative proper motions), and determined successfully the distribution parameters and membership of a region with two open clusters.

As we pointed out in the introduction, there may be two open clusters, NGC 1817 and NGC 1807, in the region examined in the present paper. In order to confirm this point, we will follow the same method to determine the distribution parameters and membership of the two open clusters.

The frequency function for the $i$-th star of a cluster can be written as follows:

$$
\begin{aligned}
\Phi_{\mathrm{c}}^{v}= & \frac{1}{2 \pi\left(\sigma_{\mathrm{c}}^{2}+\epsilon_{x i}^{2}\right)^{1 / 2}\left(\sigma_{\mathrm{c}}^{2}+\epsilon_{y i}^{2}\right)^{1 / 2}} \\
& \exp \left\{-\frac{1}{2}\left[\frac{\left(\mu_{x i}-\mu_{x c}\right)^{2}}{\sigma_{\mathrm{c}}^{2}+\epsilon_{x i}^{2}}+\frac{\left(\mu_{y i}-\mu_{y c}\right)^{2}}{\sigma_{\mathrm{c}}^{2}+\epsilon_{y i}^{2}}\right]\right\},
\end{aligned}
$$

where $\left(\mu_{x i}, \mu_{y i}\right)$ are the proper motions of the $i$-th star, $\left(\mu_{x \mathrm{c}}, \mu_{y \mathrm{c}}\right)$ the cluster proper motion center, $\sigma_{\mathrm{c}}$ the intrinsic proper motion dispersions of member stars and 


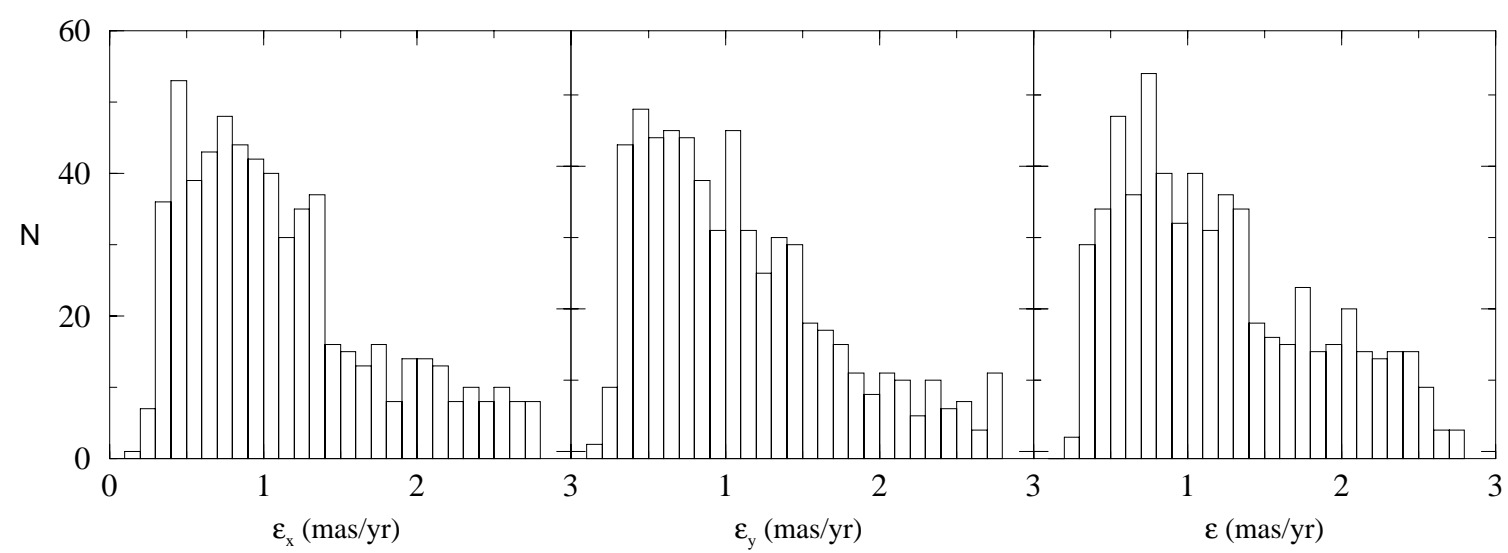

Fig. 2. The rms errors of proper motions vs. the number of stars

$\left(\epsilon_{x i}, \epsilon_{y i}\right)$ are the observed errors of the proper-motion components of the $i$-th star.

And for the field,

$$
\begin{gathered}
\Phi_{\mathrm{f}}^{\boldsymbol{v}}= \\
\exp \left\{-\frac{1}{2 \pi\left(1-\gamma^{2}\right)^{1 / 2}\left(\sigma_{x \mathrm{f}}^{2}+\epsilon_{x i}^{2}\right)^{1 / 2}\left(\sigma_{y \mathrm{f}}^{2}+\epsilon_{y i}^{2}\right)^{1 / 2}}\left[\frac{\left(\mu_{x i}-\mu_{x \mathrm{f}}\right)^{2}}{\sigma_{x \mathrm{f}}^{2}+\epsilon_{x i}^{2}}\right.\right. \\
\left.\left.-\frac{2 \gamma\left(\mu_{x i}-\mu_{x \mathrm{f}}\right)\left(\mu_{y i}-\mu_{y \mathrm{f}}\right)}{\left(\sigma_{x \mathrm{f}}^{2}+\epsilon_{x i}^{2}\right)^{1 / 2}\left(\sigma_{y \mathrm{f}}^{2}+\epsilon_{y i}^{2}\right)^{1 / 2}}+\frac{\left(\mu_{y i}-\mu_{y \mathrm{f}}\right)^{2}}{\sigma_{y \mathrm{f}}^{2}+\epsilon_{y i}^{2}}\right]\right\},
\end{gathered}
$$

where $\left(\mu_{x i}, \mu_{y i}\right)$ are the proper motions of the $i$-th star, $\left(\mu_{x \mathrm{f}}, \mu_{y \mathrm{f}}\right)$ the field proper motion center, $\left(\epsilon_{x i}, \epsilon_{y i}\right)$ are the observed errors of the proper-motion components of the $i$-th star, $\left(\sigma_{x \mathrm{f}}, \sigma_{y \mathrm{f}}\right)$ the field intrinsic proper motion dispersions and $\gamma$ the correlation coefficient.

On the other hand, the surface-number-density of cluster members is a function of the position. A Gaussian profile is the chosen approximation,

$\Phi_{\mathrm{c}}^{r}=\frac{1}{2 \pi r_{\mathrm{c}}^{2}} \cdot \exp \left\{-\frac{1}{2}\left[\left(\frac{x_{i}-x_{\mathrm{c}}}{r_{\mathrm{c}}}\right)^{2}+\left(\frac{y_{i}-y_{\mathrm{c}}}{r_{\mathrm{c}}}\right)^{2}\right]\right\}$,

where $\left(x_{\mathrm{c}}, y_{\mathrm{c}}\right)$ is the center of the cluster, and $r_{\mathrm{c}}$ the characteristic radius.

And a uniform distribution of field stars is adopted,

$\Phi_{\mathrm{f}}^{r}=\frac{1}{\pi r_{\max }^{2}}$.

The distribution of all stars in the region can, then, be described as follows:

$\Phi=\Phi_{\mathrm{c}}+\Phi_{\mathrm{f}}=\sum_{c=1}^{2} n_{\mathrm{c}} \cdot \Phi_{\mathrm{c}}^{v} \cdot \Phi_{\mathrm{c}}^{r}+n_{\mathrm{f}} \cdot \Phi_{\mathrm{f}}^{\boldsymbol{v}} \cdot \Phi_{\mathrm{f}}^{r}$.

$\sum_{c=1}^{2} n_{\mathrm{c}}+n_{\mathrm{f}}=1$.

respectively, $\Phi_{\mathrm{c}}^{r}, \Phi_{\mathrm{f}}^{r}, \Phi_{\mathrm{c}}^{v}$, and $\Phi_{\mathrm{f}}^{v}$, are the normalized distribution functions of cluster members and field stars in the position $(\boldsymbol{r})$ and relative proper motion $(\boldsymbol{v})$ spaces. With $n_{\mathrm{c}}$, the normalized number of cluster stars, and $n_{\mathrm{f}}$, the normalized number of field stars.

\section{Results and discussion}

The unknown parameters for this distribution are $\left(n_{\mathrm{c}}, x_{\mathrm{c}}, y_{\mathrm{c}}, r_{\mathrm{c}}, \mu_{x \mathrm{c}}, \mu_{y \mathrm{c}}, \sigma_{\mathrm{c}}\right)_{c=1,2}$ and $\left(n_{\mathrm{f}}, \mu_{x \mathrm{f}}, \mu_{y \mathrm{f}}, \sigma_{x \mathrm{f}}, \sigma_{y \mathrm{f}}, \gamma\right)$. Membership probabilities of the $i$-th star belonging to the $c$-th cluster can be calculated from the following,

$P_{\mathrm{c}}(i)=\frac{\Phi_{\mathrm{c}}(i)}{\Phi(i)} \quad(c=1,2)$.

According to the standard maximum likelihood method we obtained the distribution parameters and their corresponding uncertainties, given in Table 3, where the units of the proper motions and proper motion dispersions are mas/yr.

Equatorial coordinates were computed using the Tycho Catalog (ESA 1997) as reference stars. Forty stars from this catalog are in the region under study. Following Galadí-Enríquez et al. (1998), the best fitting of these reference stars was a second order pair of equations. The cross-identifications of these 40 stars are given in Table 4. Only 15 stars were found in this region from the PPM Catalog, and the respective identification is also included in the same table.

Table 5 lists the results for all 722 stars in the region of the two open clusters: Col. 1 is the ordinal star number; Cols. 2 and 3 are $\alpha_{\mathrm{J} 2000}$ and $\delta_{\mathrm{J} 2000}$ with an epoch of 1991.25; Cols. 4 and 5 are the proper motions; Cols. 6 and 7 are the standard errors of the proper motions; Cols. 8, 9 , and 10 are probabilities of stars belonging to NGC 1817 $\left(P_{1}\right)$, NGC $1807\left(P_{2}\right)$, and the field $\left(P_{\mathrm{f}}\right)$ respectively; and Col. 11 is the number of plate pairs used in the present study.

Figures 3 and 4 show the proper motion vector-point diagram and the position distribution on the sky for all the measured stars respectively, where "•" denotes a member of NGC 1817 with $P_{1} \geq 0.7$, "०" a member of NGC 1807 with $P_{2} \geq 0.7$, and all another stars are considered field stars indicated by " $\times$ ". It can be noted from the two diagrams that the centers in positional space and the centers in velocity (proper motion) space for the two open clusters 
Table 3. Distribution parameters and their uncertainties for NGC 1817 and NGC 1807. The coordinates are given in J2000, epoch 1991.25. The units of $\mu$ and $\sigma$ are in mas/yr

\begin{tabular}{|c|c|c|c|c|c|c|c|c|c|c|}
\hline & $\begin{array}{l}\text { No. } \\
\text { stars }\end{array}$ & $\begin{array}{l}\alpha_{\mathrm{J} 2000} \\
\left(5^{\mathrm{h}}+\right)\end{array}$ & $\begin{array}{c}\delta_{\mathrm{J} 2000} \\
\left(16^{\circ} .+\right)\end{array}$ & $r_{\mathrm{c}}$ & $\mu_{x}$ & $\overline{\mu_{y}}$ & $\sigma_{\mathrm{c}}$ & $\overline{\sigma_{x}}$ & $\overline{\sigma_{y}}$ & $\gamma$ \\
\hline NGC 1817 & 440 & $\begin{array}{c}12^{\mathrm{m}} 20^{\mathrm{s}} .14 \\
\quad \pm 2.3\end{array}$ & $\begin{array}{c}43^{\prime} 47^{\prime \prime} .45 \\
\pm 32^{\prime \prime}\end{array}$ & $\begin{array}{l}13.91 \\
\pm 0.55\end{array}$ & $\begin{array}{c}2.33 \\
\pm 0.22\end{array}$ & $\begin{array}{c}5.20 \\
\pm 0.21\end{array}$ & $\begin{array}{c}3.45 \\
\pm 0.10\end{array}$ & & & \\
\hline NGC 1807 & $\begin{array}{c}27 \\
\pm 4.4\end{array}$ & $\begin{array}{c}10^{\mathrm{m}} 55^{\mathrm{s}} .66 \\
\pm 4^{\mathrm{s}} .8\end{array}$ & $\begin{array}{c}23^{\prime} 23^{\prime \prime} \cdot 39 \\
\quad \pm 48^{\prime \prime}\end{array}$ & $\begin{array}{c}5.14 \\
\pm 0^{\prime \prime} 65\end{array}$ & $\begin{array}{l}-1.82 \\
\pm 0.68\end{array}$ & $\begin{array}{c}5.30 \\
\pm 0.76\end{array}$ & $\begin{array}{c}2.39 \\
\pm 0.37\end{array}$ & & & \\
\hline field & 250 & & & & -5.30 & -1.28 & & 15.0 & 14.1 & -0.045 \\
\hline
\end{tabular}

Table 4. The cross-identification of stars in Table 5 with the TYCHO and PPM catalogues

\begin{tabular}{|c|c|c|c|c|c|c|c|c|}
\hline Table 5 & TYCHO & PPM & Table 5 & TYCHO & PPM & Table 5 & TYCHO & PPM \\
\hline 48 & 12830618 & & 59 & 12831012 & & 97 & 12871643 & \\
\hline 101 & 12871461 & & 110 & 12871849 & & 137 & 12830972 & \\
\hline 140 & 12830967 & & 207 & 12871276 & 120726 & 215 & 12871736 & \\
\hline 244 & 12830546 & & 262 & 12830850 & & 290 & 12830927 & 120723 \\
\hline 297 & 12830843 & 120713 & 298 & 12830827 & & 315 & 12830675 & 120714 \\
\hline 402 & 12831299 & & 473 & 12830586 & & 476 & 12830608 & \\
\hline 527 & 12830699 & & 530 & 12830779 & 120705 & 547 & 12831086 & 120700 \\
\hline 561 & 12830896 & 120703 & 572 & 12820353 & 120694 & 576 & 12820348 & \\
\hline 653 & 12871837 & & 683 & 12820399 & & 703 & 12820600 & 120687 \\
\hline 709 & 12820398 & 120684 & 731 & 12820318 & & 767 & 12860220 & 120682 \\
\hline 797 & 12820534 & & 807 & 12820452 & 120680 & 813 & 12820504 & \\
\hline 819 & 12820541 & & 822 & 12820550 & 120673 & 825 & 12820569 & 120672 \\
\hline 827 & 12820576 & & 831 & 12820689 & & 835 & 12820586 & 120674 \\
\hline 881 & 12820253 & & & & & & & \\
\hline
\end{tabular}

are very clearly separated, which can be confirmed from the distribution parameters listed in Table 3. The dispersion of the cluster proper motions in this table is due to two reasons: the intrinsic velocity dispersion of the cluster members on one hand, and the precision of the proper motions (that in any case is poorer than usual, maybe because the epoch differences of plates are not long enough) on the other hand. The dispersion of proper motions compared to the mean internal errors seems to indicate that the velocity dispersion of members is larger than in other clusters, if the distance of $1800 \mathrm{pc}$ (HH 1997) is assumed.

The angular diameter of NGC 1817 presented here is significantly larger than the values found by previous authors. Cuffey (1938) gave an estimation of the angular diameter based on his photometric study of the central region. We found a bigger diameter based on a complete astrometric study of all the region. NGC 1817 has an halo more extended that can be seen in a visual inspeccion of the area. Further studies using photometric data and radial velocities will be very helpful on this subject.

The membership probability histogram (Fig. 5) shows a very clear separation between cluster members and field stars. We find that the numbers of stars with membership probabilities higher than 0.7 for NGC 1817 and NGC 1807 are 416 and 14 respectively, and their average membership probabilities are 0.93 and 0.83 respectively, i.e., contamination by field stars is expected to be only $7 \%$ and $17 \%$ for the two clusters. If these stars were considered as belonging to the field, the apparent deficiency on field stars in the central region should vanish.

Till this point, all of our work indicates that the determination of two open clusters is successful: there exist two real open clusters NGC 1817 and NGC 1807. But, unfortunately, the fact of the NGC 1807 cluster being near to the edge of the plates makes the number of stars measured very small for what might be desirable. Conclusive results for this cluster should come from photometry or radial velocities studies. In this sense our group is working on a complete photometric study of the region that could be highly helpful in this respect.

\subsection{Effectiveness of membership determination}

Contamination by background and foreground objects through the influence of the observational projection effect can not be avoided. Following Shao \& Zhao (1996) we can judge quantitatively how effective the results of our membership determination was. The effectiveness of membership determination is set as:

$E=1-\frac{N \sum_{i=1}^{N}\{P(i)[1-P(i)]\}}{\sum_{i=1}^{N} P(i) \sum_{i=1}^{N}[1-P(i)]}$

where the bigger $E$ is, the more effective the membership determination is. 


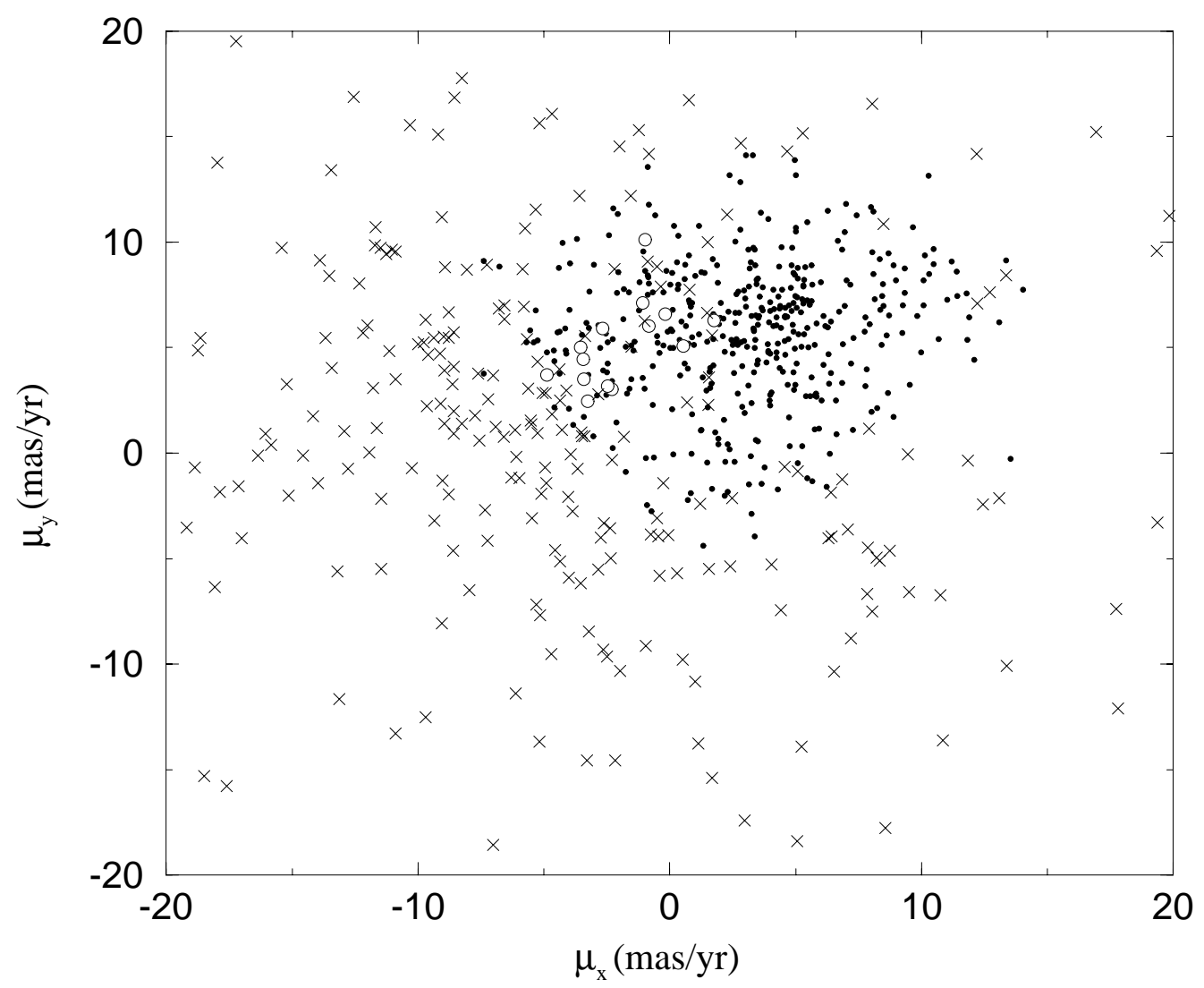

Fig. 3. The proper motion vector-point diagram of NGC 1817 and NGC 1807. ("•" denotes a member of NGC 1817 with $P_{1} \geq 0.7$, "o" a member of NGC 1807 with $P_{2} \geq 0.7$, "×" a field star)

So we can determine that the effectiveness of membership determination is 0.68 and 0.63 for NGC 1817 and NGC 1807 respectively. It is shown in Fig. 3 of Shao's paper (Shao \& Zhao 1996) that the effectiveness of membership determination of 43 open clusters are from 0.20 to 0.90 and the peak value is 0.55 . Compared with previous works (Shao \& Zhao 1996; Tian et al. 1998), we can see that the effectiveness of membership determination for the two open clusters is significantly high in both cases.

\subsection{Surface density distribution}

The surface density distribution for the cluster members can be defined by the following equations:

$\rho_{\mathrm{c}}=\frac{\sum_{i=1}^{N} P_{\mathrm{c}}(i)}{\Delta S} \pm \frac{\sqrt{\sum_{i=1}^{N} P_{\mathrm{c}}(i)}}{\Delta S}$.

The second term of the right side of the above equation is the uncertainty, $\sigma_{i}$, which follows the Poisson distribution; at the same time the surface density distribution of the field stars is:

$\rho_{\mathrm{f}}=\frac{\sum_{i=1}^{N} P_{\mathrm{f}}(i)}{\Delta S}$

In Eqs. (11) and (12) the sums are performed for the stars in the area $\Delta S$ using the membership probabilities for each of the two clusters $\left(P_{\mathrm{c}}(i), c=1,2\right)$ and the field $\left(P_{\mathrm{f}}\right)$ in turn. $\rho_{\mathrm{c}}$ and $\rho_{\mathrm{f}}$ are calculated for each different $\Delta S$, which is defined as an annulus with varying radial distance from the cluster center, and $\rho_{\mathrm{c}}$ is calculated separately for each of the two clusters. Table 6 gives the surface density distributions $\rho_{\mathrm{c}}$ of the member stars and the corresponding uncertainty $\sigma$ in the two distributions.

Figure 6 shows the radial density profiles of members of the two open clusters and of the field stars. We can see from this figure that both NGC 1817 and NGC 1807 have good central concentration, while on the other hand the surface density of field stars is quite uniform in the whole region. At the same time, this figure indicates that the two star clusters defined in the present study actually exist independently.

Acknowledgements. We would like to thank Prof. J.J. Wang, Dr. Z.Y. Shao and Dr. C.G. Su for their useful discussions. We also would like to thank Dr. C.Jordi and Dr. D.GaladíEnríquez for their kind help and useful comments on the final steps of this paper.

This work was partially supported under the National Natural Science Foundation of China Grant No. 19673012 and No. 19733001, and by the Centro de Estudios de Asia Oriental (FGUAM), Universidad Autónoma de Madrid. This work was 


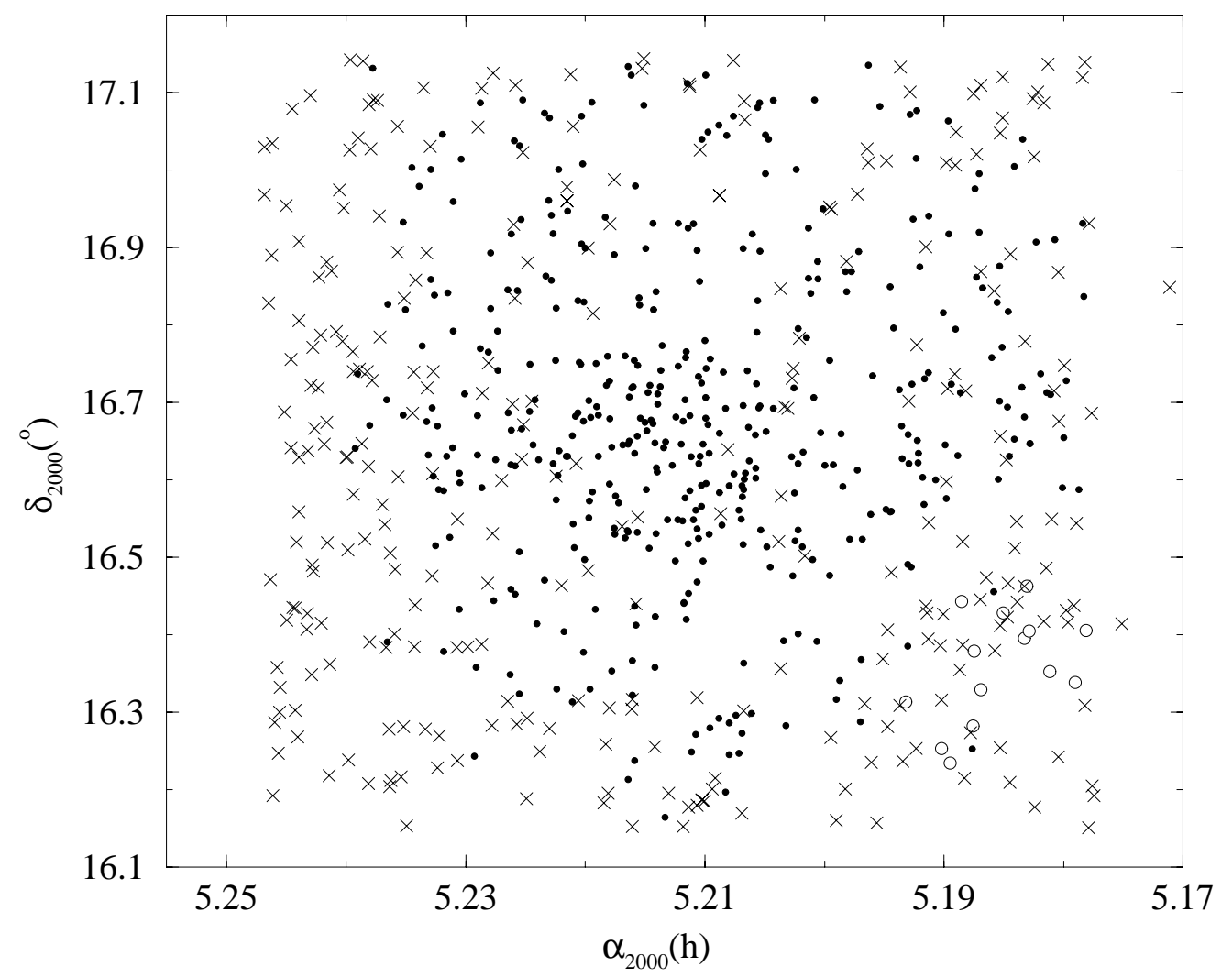

Fig. 4. The position distribution of stars in NGC 1817 and NGC 1807 area. ("•" denotes a member of NGC 1817 with $P_{1} \geq 0.7$, "o" a member of NGC 1807 with $P_{2} \geq 0.7$, "×" a field star)

Table 6. The surface densities of the member stars and the corresponding uncertainties in two open clusters

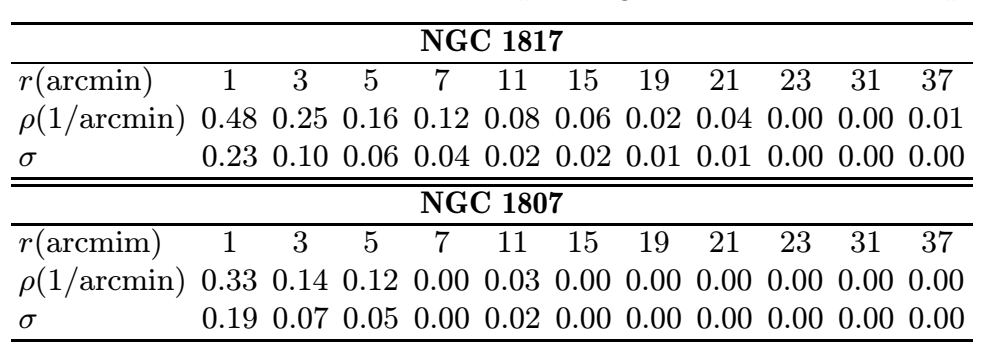

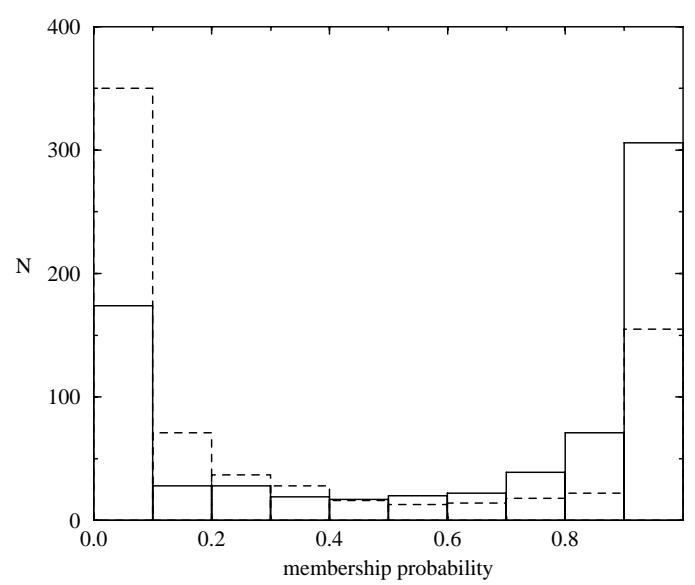

Fig. 5. The histogram of membership probability of NGC 1817 (solid line for the cluster stars, dashed line for the field stars)

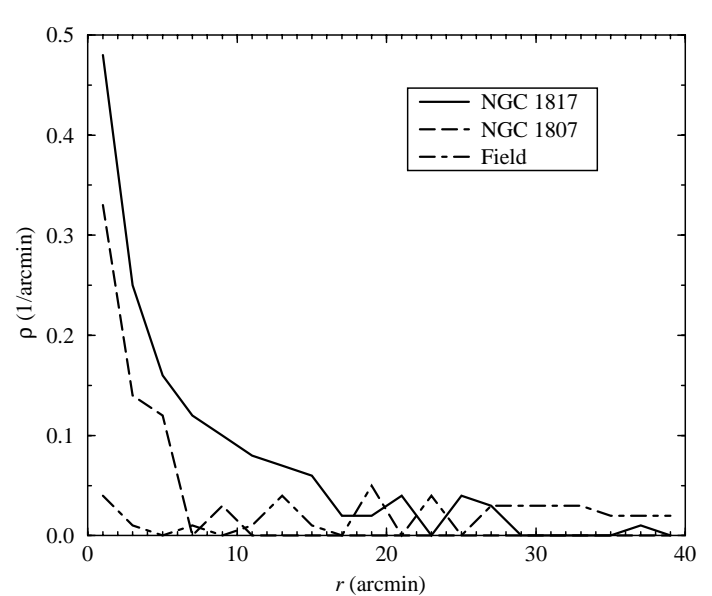

Fig. 6. The radial density profiles 
also partially supported under Joint Laboratory for Optional Astronomy of CAS.

\section{References}

Barkhatova K.A., 1963, Sbor. Rab. Astr. Ural. 1, 3

Becker W., Fenkart R., 1971, A\&AS 4, 241

Cuffey J., 1938, Ann. Harvard Obs. 106, 39

ESA, 1997, The Hipparcos and Tycho Catalogues, ESA-SP 1200

Galadí-Enríquez D., Jordi C., Trullols E., Guibert J., Tian K.P., Zhao J.L., 1998, A\&AS 131, 239

Harris G.L., Harris W.E., 1977, AJ 82, 612

Lee J.-F., van Altena W.F., 1983, AJ 88, 1683

Li H., 1954, Ann. Zǒ-Sè Obs., Nanking, Vol. 23

Janes K., Adler, D., 1982, ApJS 49, 425

Janes K.A., Phelps R.L., 1994, AJ 108, 1773

Phelps R.L., Janes K.A., Montgomery K.A., 1994, AJ 107, 1079

Piatti A.E., Claria J.J., Abadi M.G., 1995, AJ 110, 2813

Purgathofer A., 1961, Z. Astrophys. 52, 186

Purgathofer A., 1964, Ann. Univ. Sternw. Wien 26, 37
Sanders W.L., 1971, A\&A 14, 226

Shao Z.Y., Zhao J.L., 1996, Acta Astron. Sin. 37, 377

Stetson P.B., 1980, AJ 85, 387

Su C.G., Zhao J.L., Tian K.P., 1998, A\&AS 128, 255

Tian K.P., Yin M.G., Xu Z.H., Zhao J.L., 1982, Ann. Shanghai Obs. 4,17

Tian K.P., Yin M.G., Jin J.Y., Xu Z.H., 1983, Ann. Shanghai Obs. 5,136

Tian K.P., Zhao J.L., Shao Z.Y., Stetson P.B., 1998, A\&AS 131,89

Vasilevskis S., Klemola A., Preston G., 1958, AJ 63, 387

Wang J.J., Chen L., Zhao J.H., Jiang P.F., 1995, A\&AS 113, 419

Yoss K.M., Karman R.A., Hartkopf W.I., 1981, AJ 86, 36

Zhao J.H., Zhao J.L., 1994, Ann. Shanghai Obs. 15, 85

Zhao J.L., Tian K.P., Pan R.S., He Y.P., Shi H.M., 1993, A\&AS 100,243

Zhao J.L., Tian K.P., Xu Z.H., Yin M.G., 1980, Acta Astron. Sin. 22,180

Zhao J.L., He Y.P., 1990, A\&A 237, 54

Zhao J.L., Pan R.S., Huang S.N., He Y.P., 1988, Acta Astron. Sin. 29, 359

Zhao J.L., Shao Z.Y., 1994, A\&A 288, 89 